\title{
On the variation of parameters that characterize the state of a physiological system. Red blood cells as an example
}

\author{
S. Svetina ${ }^{1,2}$, M. Brumen ${ }^{2,3}$, M. Gros ${ }^{1}$, S. Vrhovec ${ }^{1} \&$ T. Žnidarčič ${ }^{1}$ \\ 'Institute of Biophysics, Faculty of Medicine, University of Ljubljana, \\ Slovenia

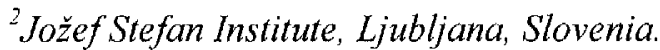 \\ ${ }^{3}$ Faculty of Education, University of Maribor, Slovenia
}

\begin{abstract}
Analysis of the population variation of parameters that characterize the state of a physiological system may provide a useful insight into its properties and behavior. To demonstrate this, we have analyzed the population variation in red blood cell $(\mathrm{RBC})$ volume, hemoglobin content, hemoglobin concentration, density, cation content and membrane area. Because of the physical and chemical laws governing the behavior of a single cell, some of these parameters are strictly interrelated. It is shown how the corresponding causal relations give rise also to relations between the parameters of the corresponding statistical distributions, i.e. standard deviations and correlation coefficients. A classification of the cell parameters treated is suggested, in the sense that RBC membrane area and hemoglobin content, which are established in the course of the cell maturation, are hierarchically at a higher level than, for instance, the cell density which is essentially defined by the instant values of hemoglobin and water content. Existing experimental data on some statistical parameters are used to derive a complete overview of $\mathrm{RBC}$ variations with regard to the cell parameters considered.
\end{abstract}

\section{Introduction}

Physiological systems in general function normally within finite ranges of values of parameters that define their state, and these ranges often serve as criteria by which to assess the state of health of the corresponding organism. In a more 


\section{Simulations in Biomedicine $V$}

basic sense, the question can be posed as to how different subjects in a population, all carrying the same genotype, may differ in the values of their parameters, while still behaving normally. Variation in system parameters appears to be a common feature of biological systems and therefore the elucidation of their origin is of fundamental interest. In order to understand the problems related to the variation of physiological parameters within a certain population of equivalent subjects, we have analyzed the population variation of red blood cells (RBCs). RBCs constitute a well defined physiological system and, because they are easily accessible, one of the most intensively characterized cells, both structurally and functionally. However, there are still many unsolved RBC problems [1].

The interest in $\mathrm{RBC}$ variations is practical as well as theoretical since they are a useful indicator of a set of hematological diseases. Hence, in commonly applied clinical laboratory tests [2] RBCs are examined with respect to their mean cell hemoglobin concentration and mean cell volume, and usually their $\mathrm{RBC}$ volume distribution width. More advanced blood analyzers [3] allow the variation of $\mathrm{RBC}$ population to be determined with respect to the hemoglobin concentration. However, RBCs are distributed also with respect to many other properties, for instance the distribution of cation content [4] and membrane area [5]. Several methods allow concomitant determination of two or more RBC properties, thus providing correlations between different properties. For example, simultaneous determination of cell volume and membrane area, by photographing the cells hanging on edge [5], have demonstrated a strong correlation between the two. Using microphotometric methods, evidence has been obtained [6] for a correlation of RBC hemoglobin content with cell surface area. Electron probe determination of the presence of different elements in single RBCs have shown a correlation between the content of univalent cations and of iron (i.e. hemoglobin) [4].

In this contribution we aim to demonstrate the information that can be obtained about a physiological system by studying, not only the mean values of a certain set of measurable parameters, but also their distribution functions. Assuming that the measurable parameters are, to a first approximation, distributed according to a Gaussian joint distribution function, the variational behavior of a system is defined by the corresponding standard deviations and correlation coefficients. In this case, when there are $\mathrm{n}$ independent parameters, the system is defined by $\mathrm{n}$ mean values, $\mathrm{n}$ standard deviations, and $\mathrm{n}(\mathrm{n}-1) / 2$ correlation coefficients, a total of $n+n(n+1) / 2$ values. However, these values may not be independent, because cells are governed by physical and chemical laws that may result in causal relations between some of the parameters [7]. Thus, if there are $r$ causal relations interrelating the system parameters, there are then only $n-r$ independent mean values, $n-r$ independent standard deviations, and ( $\mathrm{n}$ $r)(n-r-1) / 2$ independent correlation coefficients. Consequently, it is possible to derive, if the causal relations can be expressed in a linear fashion, $r(2 n-r+1) / 2$ relations between the standard deviations and correlation coefficients pertaining to the $n$ system parameters [7].

In the previous work [7], we analyzed the relations between the variation of human RBC volume, density, membrane area, hemoglobin content and cation 
content. One causal relation between these parameters is the dependence of cell density on the hemoglobin content and the cell volume. Another causal relation is the osmotically governed dependence of the cell volume on hemoglobin and cation content $[8,9]$. On the basis of these two causal relations valid for a single cell $(r=2)$, it was possible to predict, from the five standard deviations of five measured parameters and three known correlation coefficients, the seven remaining correlation coefficients and the two coefficients expressing the causal linearized dependence of the cell volume on hemoglobin and cation content. The predicted values were consistent with the measured mean values of hemoglobin and cation content of density fractionated RBC subpopulations [10]. The analysis also showed a statistical correlation between hemoglobin content and membrane area which indicated, for a single cell, a strict dependence of cation content on these two parameters. This insight has gained additional support from an analysis showing that, with respect to at least some membrane constituents, membranes in a RBC population do not vary appreciably [11].

The analyses described could only be made [7] on the basis of data obtained in different laboratories, applying different sample preparations and using different methods. Over the last twenty years many new experimental approaches have been introduced which now make it possible to reexamine the above conclusions and to extend the analysis to other RBC properties. The new data allow a more systematic approach because the analysis can be performed separately with regard to the expression for the cell density (and for the related definition of hemoglobin concentration) and with regard to the expression describing the RBC osmotic state. The studies particularly suitable for the purposes of our analyses are those of Mohandas [12], Lew [13] and Lee [4]. The former applied laser light scattering to make accurate and independent measurements of volume and hemoglobin concentration on individual RBCs. This allows relations to be obtained between population variation of cell volume, hemoglobin concentration, hemoglobin content and cell density. Lew [13] added measurement of osmotic lysis, allowing the statistical proportionalities between cell osmolyte content, hemoglobin content, and membrane area to be recognized. They addressed the question as to whether the distributions of volume, area, hemoglobin content and hemoglobin concentration observed in human RBCs depend on regulated processes operating throughout cell maturation, or rather on the distribution of relevant properties at the last erythroid cell division. Their analysis showed the possibility of the latter explanation. Lee [4] studied interrelations in human $\mathrm{RBC}$ populations between the $\mathrm{Na}$ and $\mathrm{K}$ contents, hemoglobin content, cell volume, and buoyant density.

The presentation will be organized as follows. In the next section we shall present the necessary mathematical expressions. Then we shall analyze the relations between variations in RBC volume, hemoglobin concentration, hemoglobin content and cell density. The following section will deal with the consequences of the osmotic state of RBCs and enable extension of the results of the previous section by including the cell osmolyte content. Finally, the effects on the RBC population of variation in membrane area will be analyzed. 


\section{Simulations in Biomedicine $V$}

\section{Joint distribution function and relevant mathematical definitions}

The joint distribution function on variables $x_{i}, i=1, \ldots, n$, is defined [14] as a probability density function $\mathrm{g}\left(\mathrm{x}_{1}, \ldots, \mathrm{x}_{\mathrm{n}}\right)$ define such that

$$
\int \mathrm{g}\left(\mathrm{x}_{1}, \cdots, \mathrm{x}_{n}\right) \mathrm{dx_{1 }} \cdots \mathrm{dx_{n }}=1
$$

If the joint probability density function on $\mathrm{n}$ variables is normal (Gaussian), it is fully described by $n$ mean values $<\mathrm{x}_{\mathrm{i}}>$, $\mathrm{n}$ standard deviations $\sigma_{\mathrm{i}}$ and $\mathrm{n}(\mathrm{n}-1) / 2$ correlation coefficients $\rho_{i, j}$, where

$$
\begin{gathered}
\quad<x_{i}>=\int x_{i} g\left(x_{1}, \cdots, x_{n}\right) d x_{1} \cdots d x_{n}, \\
\sigma_{i}^{2}=\int\left(x_{i}-<x_{i}>\right)^{2} g\left(x_{1}, \cdots, x_{n}\right) d x_{1} \cdots d x_{n},
\end{gathered}
$$

and

$$
\rho_{\mathrm{i}, \mathrm{j}} \sigma_{i} \sigma_{\mathrm{j}}=\int\left(\mathrm{x}_{\mathrm{i}}-<\mathrm{x}_{\mathrm{i}}>\right)\left(\mathrm{x}_{\mathrm{j}}-<\mathrm{x}_{\mathrm{j}}>\right) \mathrm{g}\left(\mathrm{x}_{1}, \cdots, \mathrm{x}_{\mathrm{n}}\right) \mathrm{dx_{1 }} \cdots \mathrm{dx}_{\mathrm{n}} .
$$

An assessment of the relations between the parameters of a cell can also be obtained from fractionation experiments. When a sample of RBCs is fractionated according to a variable $\mathrm{x}_{\mathrm{i}}$, each fraction can determine the mean of any variable $x_{j}, j \neq i$. For the theoretical determination of this mean it is sufficient to use the joint distribution function with two variables $g\left(x_{i}, x_{j}\right)$ where

$$
g\left(x_{i}, x_{j}\right)=\int g\left(x_{1}, \cdots, x_{n}\right) d x_{1} \cdots d x_{i-1} d x_{i+1} \cdots d x_{j-1} d x_{j+1} \cdots d x_{n}
$$

The mean value of variable $x_{j}$ within the $l$-th fraction, $F_{i, \ell}$ is a conditional mean $<\mathrm{x}_{\mathrm{j}} \mid \mathrm{x}_{\mathrm{i}} \in \mathrm{F}_{\mathrm{i}, \mathrm{l}}>$ which can be expressed as

$$
<\mathrm{x}_{\mathrm{j}} \mid \mathrm{x}_{\mathrm{i}} \in \mathrm{F}_{\mathrm{i}, \ell}>=<\mathrm{x}_{\mathrm{j}}>+\frac{\sigma_{\mathrm{j}}}{\sigma_{\mathrm{i}}} \rho_{\mathrm{i}, \mathrm{j}}\left(<\mathrm{x}_{\mathrm{i}} \mid \mathrm{x}_{\mathrm{i}} \in \mathrm{F}_{\mathrm{i}, \ell}>-<\mathrm{x}_{\mathrm{i}}>\right) \text {. }
$$

The fraction mean of $x_{j}$ is proportional to the fraction mean of $x_{i}$ where the constant of proportionality is determined by the corresponding standard deviations and correlation coefficient.

In order to show how strict relations between the cell parameters lead to relations between the statistical parameters, let us assume that the variable $x_{k}$ can be causally expressed as a linear function of $m$ variables $x_{i}$

$$
\mathrm{x}_{\mathrm{k}}=<\mathrm{x}_{\mathrm{k}}>+\sum_{\mathrm{i}=1}^{\mathrm{m}} \mathrm{a}_{\mathrm{k}, \mathrm{i}}\left(\mathrm{x}_{\mathrm{i}}-<\mathrm{x}_{\mathrm{i}}>\right),
$$

where $a_{k, i}$ are constants. The relations between statistical parameters are then

$$
\sigma_{k}^{2}=\sum_{i=1}^{m} \sum_{j=1}^{m} a_{k, i} a_{k, j} \sigma_{i} \sigma_{j} \rho_{i, j},
$$

and

$$
\sigma_{k} \rho_{k, i}=\sum_{j=1}^{m} a_{k, j} \sigma_{j} \rho_{i, j}
$$




\section{Relations between variation in $R B C$ volume, hemoglobin content, hemoglobin concentration and cell density}

Variations in RBC volume (V), hemoglobin content $(\mathrm{H})$, hemoglobin concentration (C), and cell density (D) are considered. There are two strict relations between these cell properties that hold for each cell. The first is the expression for hemoglobin concentration:

$$
\mathrm{C}=\frac{\mathrm{H}}{\mathrm{V}} \text {, }
$$

and the second is the expression for RBC density, obtained by assuming that hemoglobin accounts for most of the red cell dry mass:

$$
\mathrm{D}=\frac{\mathrm{H}+\mathrm{D}_{\mathrm{s}} \mathrm{V}_{\mathrm{s}}}{\mathrm{V}}=\mathrm{D}_{\mathrm{s}}+\left(1-\frac{\mathrm{D}_{\mathrm{s}}}{\mathrm{D}_{\mathrm{H}}}\right) \frac{\mathrm{H}}{\mathrm{V}},
$$

where $V_{s}$ is the volume of the RBC water content, $D_{H}$ the hemoglobin density and $D_{\mathrm{s}}$ the density of the water solution $\left(D_{H} \approx 1.36 \mathrm{~g} / \mathrm{ml}\right.$ and $\left.D_{\mathrm{s}} \approx 1 \mathrm{~g} / \mathrm{ml}\right)$. It is clear from eqs (10) and (11) that hemoglobin concentration and cell density are strictly related. However, both properties are of interest because they can both be measured experimentally.

With four parameters $(n=4)$ and two strict relations $(r=2)$, there are three independent statistical parameters and seven relations between them. Because of the proportionality between $\mathrm{D}$ and $\mathrm{C}$, eq (11), we have

$$
\sigma_{D}=\left(1-\frac{D_{s}}{D_{H}}\right) \sigma_{C}
$$

and the correlation coefficient $\rho_{D, C}$ equals 1 . It follows also that correlation coefficients between density and either volume or hemoglobin content are the same as between hemoglobin concentration and the same cell parameters, $\rho_{\mathrm{C}, \mathrm{i}}=$ $\rho_{D, i}(i=V, H)$. For the complete characterization of this system we thus need three independent measurements and three additional relations.

Our analysis is based on the experimental data of Mohandas [12] who measured $\mathrm{C}$ and $\mathrm{V}$ of individual cells and, in a separate experiment, the mean values of $\mathrm{C}$ and $\mathrm{V}$ of density fractionated RBCs. We assume that the data in their Fig. 3 corresponds to that in their Table 1 . In this analysis it is useful to consider $\mathrm{C}$ and $\mathrm{V}$ as independent variables. Therefore we re-express eq (10) as $\mathrm{H}=\mathrm{CV}$. In order to obtain a linear dependence of $\mathrm{H}$ on $\mathrm{C}$ and $\mathrm{V}$ of the type of eq (7), $\mathrm{H}$ is expanded up to the first order terms around its mean value $\langle\mathrm{H}\rangle=\langle\mathrm{C}\rangle\langle\mathrm{V}\rangle$, where $\langle\mathrm{C}\rangle$ and $\langle\mathrm{V}\rangle$ are the mean values of $\mathrm{C}$ and $\mathrm{V}$, respectively:

$$
\mathrm{H}=\langle\mathrm{H}\rangle+\langle\mathrm{V}\rangle(\mathrm{C}-\langle\mathrm{C}\rangle)+\langle\mathrm{C}\rangle(\mathrm{V}-\langle\mathrm{V}\rangle) \text {. }
$$

By applying eqs (8) and (9), the three relations required to determine $\sigma_{\mathrm{H}}, \rho_{\mathrm{H}, \mathrm{C}}$ and $\rho_{\mathrm{H}, \mathrm{V}}$ are:

$$
\begin{gathered}
\sigma_{H}^{2}=\langle V\rangle^{2} \sigma_{C}^{2}+2\langle V\rangle\langle C\rangle \sigma_{C} \sigma_{V} \rho_{C, V}+\langle C\rangle^{2} \sigma_{V}^{2}, \\
\sigma_{H} \rho_{H, C}=\langle V\rangle \sigma_{C}+\langle C\rangle \sigma_{V} \rho_{V, C},
\end{gathered}
$$

and 


\section{Simulations in Biomedicine $V$}

Table 1. The coefficients of variation $\left(\sigma_{i} /<x_{i}>\right)$ (diagonal elements) and correlation coefficients (non-diagonal elements) related to $\mathrm{RBC}$ volume, hemoglobin content, hemoglobin concentration, cell density, cation content and membrane area. The table should be read, in accord with the text in this and the following two sections, for the first four, five and six parameters, respectively. Experimental values are presented in bold letters and calculated values in regular print. The experiments from which data are taken and the methods of calculation are presented in the text.

\begin{tabular}{|c|c|c|c|c|c|c|}
\hline & V & $\mathrm{H}$ & $\mathrm{C}$ & $\bar{D}$ & $\mathrm{~K}$ & $\bar{A}$ \\
\hline V & 0.132 & 0.89 & -0.50 & -0.50 & 0.82 & 0.97 \\
\hline $\mathrm{H}$ & & 0.112 & -0.04 & -0.04 & 0.47 & 0.73 \\
\hline C & & & 0.061 & 1 & -0.90 & -0.73 \\
\hline D & & & & 0.005 & -0.90 & -0.73 \\
\hline $\mathrm{K}$ & & & & & 0.205 & 0.96 \\
\hline $\mathrm{A}$ & & & & & & 0.121 \\
\hline
\end{tabular}

$$
\sigma_{\mathrm{H}} \rho_{\mathrm{H}, \mathrm{V}}=\langle\mathrm{V}\rangle \sigma_{\mathrm{C}} \rho_{\mathrm{V}, \mathrm{C}}+\langle\mathrm{C}\rangle \sigma_{\mathrm{V}}
$$

The coefficients of variation (standard deviations/mean values) and correlation coefficients for the four considered RBC parameters are presented in the first four columns of Table 1 . The mean values $\langle\mathrm{C}\rangle$ and $\langle\mathrm{V}\rangle$ and standard deviations $\sigma_{C}$ and $\sigma_{V}$ are taken from Mohandas [12], as is the correlation coefficient $\rho_{C, V}=$ $\rho_{C, D}$ which is obtained by equating the slope, in their density fractionation measurement, of the dependence of fraction mean volume on fraction mean hemoglobin concentration (Fig. 1), with the slope in the corresponding eq (6). The remaining statistical parameters are obtained from eqs (14) to (16).

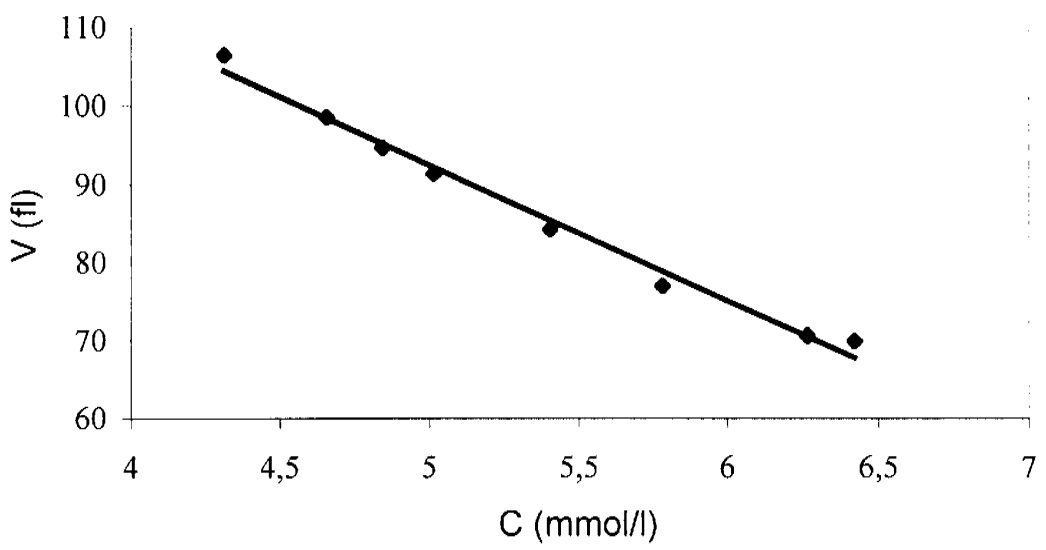

Figure 1: The dependence of fraction mean volume (V) on fraction mean hemoglobin concentration (C) taken from Table 1 of Mohandas [12]. 
Table 1 shows that the distribution widths regarding different parameters are different. The width of the distribution with respect to hemoglobin concentration is half narrower than the distributions for the volume and hemoglobin content, whereas distribution with respect to density is even narrower. Volume and hemoglobin are strongly correlated whereas there is a rather small correlation between hemoglobin content and density. Dense cells have slightly less hemoglobin than lighter cells. Such behavior was also documented in [4], whereas the data of Lew [13] indicate the opposite effect, with the correlation coefficient 0.12 .

\section{Relations between variation of $\mathrm{RBC}$ properties resulting from RBC osmotic behavior}

The volume of an individual RBC is essentially the sum of the volume of hemoglobin molecules in the cell and its water content. Water can easily flow through the RBC membrane and adjust its content to establish osmotic equilibrium between the outside and inside of the cell. In RBCs it is mainly the amount of the cell cations and hemoglobin which determine osmotically the cell water content. Since the cell membrane cannot resist any osmotic pressure difference across the membrane the osmotic pressure of the inner cell solution is equal to the osmotic pressure of the external solution.

It is well known that, to describe the osmotic equilibrium in $\mathrm{RBCs}$, the equilibria of chloride and hydroxyl ions have to be considered. The transport mechanisms of chloride and hydroxyl ions are complex and usually fast with respect to days and hours, so the equilibrium of these ions can be considered in terms of the corresponding Donnan equilibrium. Furthermore, the electroneutrality condition of the inner cell solution is an important constraint for the distribution of chloride and hydroxyl ions across the membrane. Hemoglobin, as both an inner solute and a buffer, has an important role in modulating the inner osmotic pressure by the concentration dependent osmotic coefficient, as well as in modulating the distribution of ions across the membrane by its $\mathrm{pH}$ dependent average charge.

The content of sodium and potassium ions in the $\mathrm{RBC}$ is maintained by the counterbalanced ionic fluxes across the cell membrane which, in terms of modeling, can be combined into $\mathrm{Na} / \mathrm{K}$-exchange active transport and the corresponding opposite leak fluxes. The characteristic time scale for these processes is known to be extremely large, so the total sodium and potassium content in the cell can be taken as being constant over hours or even days. Other ions have no significant influence on the osmotic behavior of RBCs.

These RBC properties can be expressed in terms of the corresponding mathematical model $[8,9]$. Its basic frame consists of equations expressing the osmotic equilibrium of water and the electroneutrality of the inner cell solution. The equilibrium of chloride and hydroxyl ions is expressed by the Nernst equation. The hemoglobin osmotic coefficient, as a function of hemoglobin concentration, and the inner $\mathrm{pH}$ dependence of the average hemoglobin charge, are taken into account in terms of experimentally obtained dependencies. In view 
of the dependent and independent cell parameters discussed above, the hemoglobin content and the total cation content are independent cell parameters, and the cell volume (i.e. the cell water volume), inner chloride concentration, inner $\mathrm{pH}$ and transmembrane electric potential difference are dependent cell parameters. External conditions such as concentration of chloride and cations, $\mathrm{pH}$ and temperature are given. In this way the model equations describe the equilibrium state of a single RBC on an appropriate time scale.

The total cell cation content is now taken into consideration as an additional determinant of the RBC state. Since the joint distribution of cation and hemoglobin content have been measured [4] it is of interest to see whether this distribution predicts reasonably the parameters describing the cell volume distribution. Furthermore, the parameters determining the equilibrium state of a single cell can be critically examined, indicating the relevance of the model equations. For this purpose, following eq (7), the cell volume can be expressed, in reasonable proximity to its mean value, as a linear function of the cation and hemoglobin content

$$
\mathrm{V}=\langle\mathrm{V}\rangle+\mathrm{a}_{1}(\mathrm{~K}-\langle\mathrm{K}\rangle)+\mathrm{a}_{2}(\mathrm{H}-\langle\mathrm{H}\rangle)
$$

Constants $a_{1}$ and $a_{2}$ can then be obtained following two different procedures. Firstly, they can be calculated directly from the equilibrium model equations. Secondly, they can be obtained from a system of equations constructed following eqs (8) and (9). In the latter approach, a system of four equations can be formulated:

$$
\begin{gathered}
\sigma_{\mathrm{V}}^{2}=\mathrm{a}_{1}^{2} \sigma_{\mathrm{K}}^{2}+2 \mathrm{a}_{1} \mathrm{a}_{2} \sigma_{\mathrm{K}} \sigma_{\mathrm{H}} \rho_{\mathrm{K}, \mathrm{H}}+\mathrm{a}_{2}^{2} \sigma_{\mathrm{H}}^{2}, \\
\sigma_{\mathrm{V}} \rho_{\mathrm{K}, \mathrm{V}}=\mathrm{a}_{1} \sigma_{\mathrm{K}}+\mathrm{a}_{2} \sigma_{\mathrm{H}} \rho_{\mathrm{K}, \mathrm{H}}, \\
\sigma_{\mathrm{V}} \rho_{\mathrm{H}, \mathrm{V}}=\mathrm{a}_{1} \sigma_{\mathrm{K}} \rho_{\mathrm{K}, \mathrm{H}}+\mathrm{a}_{2} \sigma_{\mathrm{H}}, \\
\sigma_{\mathrm{V}} \rho_{\mathrm{V}, \mathrm{D}}=\mathrm{a}_{1} \sigma_{\mathrm{K}} \rho_{\mathrm{K}, \mathrm{D}}+\mathrm{a}_{2} \sigma_{\mathrm{H}} \rho_{\mathrm{H}, \mathrm{D}},
\end{gathered}
$$

in which the constants $a_{1}$ and $a_{2}$ and the correlation coefficients $\rho_{K, V}$ and $\rho_{K, D}$ are the four unknown parameters. We take the values of $\sigma_{K}$ and $\rho_{K, H}$ from experiment [4]. These, and the obtained values of standard deviation and correlation coefficients, are presented in the fifth column of Table 1 . The values of constants $a_{1}$ and $a_{2}$ are given in Table 2 . The obtained values are similar to the values obtained previously [7], however, the discrepancy between the model prediction and the variation determination of the coefficients $a_{1}$ and $a_{2}$ is smaller.

Table 2. Constants $a_{1}$ and $a_{2}$ obtained (A) from model equations for the equilibrium state of a single cell and (B) by analysis of the relations between the variational parameters.

\begin{tabular}{|cc|c|c|}
\hline & & $\mathrm{A}$ & $\mathrm{B}$ \\
\hline $\mathrm{a}_{1}$ & $\mathrm{fl} / \mathrm{fmol}$ & 5.4 & 3.08 \\
$\mathrm{a}_{2}$ & $\mathrm{fl} / \mathrm{fmol}$ & 71 & 137 \\
\hline
\end{tabular}




\section{Effects of population variation on RBC membrane area}

RBCs also vary in the area of their membranes. The statistical link between variation in membrane area and the above treated $\mathrm{RBC}$ parameters has been demonstrated by Canham and Burton [5] who performed single cell measurements of the cell volume and membrane area. The correlation between these two parameters is strong, $\rho_{A, V}=0.97$. Another indication of such a link is the reported [6] correlation between the membrane area and the hemoglobin content, yielding a correlation coefficient of 0.73 . Lew [13] in their analysis, taking into consideration the fact that variation in membrane area affects the osmotic lysis curve, came to the analogous conclusion that the membrane area correlates with the cell cation and hemoglobin content.

The unknown correlation coefficients between the membrane area and the previously considered RBC parameters are obtained from the measured standard deviation for the area [5] and the membrane area/volume [5] and the membrane area/hemoglobin [6] correlation coefficients. The results are presented in the sixth column of Table 1. The predicted correlation coefficients have reasonable values.

The osmotic lysis curve is a further $\mathrm{RBC}$ property that can be related to the membrane area. In this curve, the fraction of lysed cells is presented as a function of tonicity (or the related salt concentration) of the external solution. The lytic tonicity depends both on the osmotic properties of the cell and on the membrane area. It can be expressed as

$$
\pi=\langle\pi\rangle+\mathrm{a}_{6}(\mathrm{~K}-\langle\mathrm{K}\rangle)+\mathrm{a}_{7}(\mathrm{H}-\langle\mathrm{H}\rangle)+\mathrm{a}_{8}(\mathrm{~A}-\langle\mathrm{A}\rangle),
$$

where the coefficients $a_{6}$, $a_{7}$ and $a_{8}$ are obtained from the requirement that the lytic concentration corresponds to the tonicity at which the cell volume predicted by the osmotic model equals that of a sphere with area $\mathrm{A}$. There are no new parameters involved, so all the statistical parameters related to the osmotic lysis curve can be determined from the previous statistical parameters. For instance, the obtained coefficient of variation for the lytic concentration is approximately equal to 0.05 , and this value is in accord with the usually observed narrow range of lytic concentrations in the osmotic fragility experiments.

\section{Discussion}

The analysis of population variation in the parameters of a physiological system is of interest, because it can (i) reveal unknown strict relations between these parameters, (ii) contribute to elucidating the processes involved during the maturation and life span of a physiological system, and (iii) may have practical applicability in revealing differences between normal and pathological states of the organisms. The discussion will follow these lines.

Some single cell relations between the parameters of a cell are obvious. It is for instance clear that the hemoglobin concentration in a given $\mathrm{RBC}$ and its density depend solely on the hemoglobin content and the volume of this cell. It is also clear that the water content of a given cell depends on the permeability properties of its membrane and is determined by the corresponding osmotic 
behavior of the cell, There is thus a set of cell parameters which are interrelated because of these two relations, as was discussed in the previous sections. The question arises about further possible relations between cell parameters arising from the physical and chemical laws that govern RBC behavior. The first parameter to consider is the cell cation content. This is the result of membrane permeability for cations, which depends on a set of passive and active transport processes. Thus, any model that would predict RBC cation content must involve the relevant properties of the membrane. The previous analysis of $\mathrm{RBC}$ variation indicated that the cation content may depend on the hemoglobin content and membrane area, because the scatter coefficient of the joint distribution function $\mathrm{g}(\mathrm{K}, \mathrm{H}, \mathrm{A})$ was found to be close to zero [7]. The present results are in accord with this notion. There is an interesting implication that membranes in different cells in a population do not differ appreciably with regard to the content of their constituents responsible for cation permeability. This property of the RBC membrane has been indicated for several other of its characteristic components such as the cholesterol/phospholipid ratio and water permeability [11].

In the search for the origin of the variations in $\mathrm{RBC}$ properties it has to be recognized that different parameters affect the behavior of the system in different ways. In the causal relations they can be divided into basic and dependent parameters. For instance, in the expression for cell density, the basic parameters are hemoglobin and water content, from which the density can be derived at any instant. The cell water content, and consequently cell volume, are dependent parameters because they have to correspond to the cell cation and hemoglobin content. The time scale within which the cell water assumes the required value is, in this case, finite because under perturbed conditions there is a finite relaxation time needed to attain the new equilibrium state. Some of the RBC parameters, such as membrane area and hemoglobin content, can be assumed to be essentially determined by the processes of RBC maturation [15], and are proportionately reduced during the 120 days of the RBC life span [16]. It can be reasoned that, because of different related relaxation times, the parameters of the $\mathrm{RBC}$ can be classified into different hierarchical levels. The parameters which are practically constant throughout the RBC life span are hierarchically at a higher level than those which can alter their value within seconds or minutes. Among the parameters we considered, membrane area and hemoglobin content are at a higher hierarchical level than the cation content, which again is higher than the cell water and volume. It can be deduced that the same holds for the statistical parameters, i.e. the standard deviations and correlation coefficients. Those for the hemoglobin content and membrane area distribution functions are basic statistical parameters, and the statistical parameters involving cell parameters of lower hierarchical level can be derived from them.

The diagnostic uses of RBC variations can benefit from the link between structural malformations and consequent alterations in the system's macroscopic behavior. The analysis presented here should help in selecting the most relevant parameters to be used in different diseases for a sensitive index of the deviations of an organism from the normal state.

In conclusion, the principles of the analysis of variational properties of physiological systems presented here may have general applicability, in 
particular because the functional studies of these systems based on knowing the corresponding genome structures, will certainly lead to better understanding about how the physiological systems are defined at the molecular level, and which laws govern their behavior at the level of individual subjects.

\section{References}

[1] Hoffman, J.F. Questions for red blood cell physiologists to ponder in this millenium. Blood Cells, Molecules, Diseases, 27(1), pp. 57-61, 2001.

[2] Thomas, L. Erythrocytes. (Chapter 16.2). Clinical Laboratory Diagnostics, ed. L. Thomas, TH-Books Verlagsgesellschaft mbH, Frankfurt/Main, pp. 470-475, 1998.

[3] Tycko, D.H., Metz, M.H., Epstein, E.A. \& Grinbaum, A. Flow-cytometric light scattering measurement of red blood cell volume and hemoglobin concentration. Appl. Optics, 24(9), pp.1355-1365, 1985.

[4] Lee, P., Kirk, R.G. \& Hoffman, J.F. Interrelations among $\mathrm{Na}$ and $\mathrm{K}$ content, cell volume, and buoyant density in human red blood cell populations. $J$. Membrane Biol., 79(2), pp. 119-126, 1984.

[5] Canham, P.B. \& Burton, A.C.: Distribution of size and shape in populations of normal human red cells. Circ. Res., 22, pp. 405-422, 1968.

[6] Heimpel, H., Kratt, E., Schwarz, J. \& Beneke G. Hämoglobingehalt und Hämoglobinverteilung in peripheren menschlichen Erythrozyten. Untersuchungen mit dem Scanning-Zytophotometer. Klin. Wochenschr., 55(23), pp. 1149-1157, 1977.

[7] Svetina, S. Relations among variations in human red cell volume, density, membrane area, hemoglobin content and cation content. J. theor. Biol., 95(1), pp. 123-134, 1982.

[8] Brumen, M., Glaser, R. \& Svetina, S. Osmotic states of the red blood cell. Bioelectroch. Bioener., 6(2), pp. 227-241, 1979.

[9] Freedman, J.C. \& Hoffman, J.F. Ionic and osmotic equilibria of human red blood-cells treated with nystatin. J. Gen. Physiol., 74(2), pp. 157-185, 1979.

[10] Cohen, N.S., Ekholm, J.E., Luthra, M.G. \& Hanahan, D.J. Biochemical characterization of density-separated human erythrocytes. Biochim. Biophys. Acta, 419(2), pp. 229-242, 1976.

[11] Svetina, S. On cell population variations of red blood cell membrane properties. Proc. of the $6^{\text {th }}$ School on Biophysics of Membrane Transport, eds.J. Kuczera, C. Grygorczyk \& S. Przestalski, The Agricultural University of Wroclaw, Wroclaw, pp. 295-310, 1981.

[12] Mohandas, N., Kim, Y.R., Tycko, D.H., Orlik, J., Wyatt, J. \& Groner W. Accurate and independent measurement of volume and hemoglobin concentration of individual red cells by laser light scattering. Blood, 68(2), pp. 506-513, 1986.

[13] Lew, V.L., Raftos, J.E., Sorette, M., Bookchin, R.M. \& Mohandas, N. Generation of normal human red cell volume, hemoglobin content, and membrane area distributions by "Birth" or regulation? Blood, 86(1), 334$341,1995$. 


\section{Simulations in Biomedicine $V$}

[14] Korn, G.A. \& Korn, T.M. Mathematical Handbook for Scientists and Engineers. McGraw-Hill Book Co., New York, Toronto, London: pp. 538 , 1961.

[15] Mohandas, N. \& Groner, W. Cell membrane and volume changes during red cell development and aging. Ann. N. Y. Acad. Sci., 554, pp.217-224, 1989.

[16] Waugh, R.E., Mohandas, N., Jackson, C.W., Mueller, T.J., Suzuki, T. \& Dale, G.L. Rheologic properties of senescent erythrocytes: Loss of surface area and volume with red blood cell age. Blood, 79(5), pp.1351-1358, 1992. 